\title{
Ginette Paquet
}

Ministère de la Santé et des Services sociaux ;

(1994)

\section{"Facteurs sociaux \\ de la santé, de la maladie \\ et de la mort”}

Un document produit en version numérique par Jean-Marie Tremblay, bénévole, professeur de sociologie au Cégep de Chicoutimi

Courriel : jean-marie tremblay@uqac.ca

Site web pédagogique : http ://www.uqac.ca/jmt-sociologue/

Dans le cadre de : "Les classiques des sciences sociales" Une bibliothèque numérique fondée et dirigée par Jean-Marie Tremblay, professeur de sociologie au Cégep de Chicoutimi Site web : http ://classiques.uqac.ca/

Une collection développée en collaboration avec la Bibliothèque Paul-Émile-Boulet de l'Université du Québec à Chicoutimi Site web : http ://bibliotheque.uqac.ca/ 
Cette édition électronique a été réalisée par Jean-Marie Tremblay, bénévole, professeur de sociologie au Cégep de Chicoutimi à partir de :

Ginette Paquet,

“Facteurs sociaux de la santé, de la maladie et de la mort”.

Un article publié dans l'ouvrage sous la direction de in ouvrage sous le direction de Fernand Dumont, Simon Langlois et Yves Martin, Traité des problèmes sociaux. Chapitre 11, pages 223 à 244. Québec: Institut québécois de recherche sur la culture, 1994, 1164 pages.

[Mme Paquet nous accordait, le 18 septembre 2007, sa permission de diffuser cet article, en texte intégral, dans Les Classiques des sciences sociales.]

1. Courriels : $\begin{aligned} & \text { ginette.paquet@inspq.qc.ca } \\ & \text { ginette.paquet@msss.gouv.qc.ca }\end{aligned}$

Polices de caractères utilisée :

Pour le texte : Times New Roman, 14 points.

Pour les citations : Times New Roman, 12 points.

Pour les notes de bas de page : Times New Roman, 12 points.

Édition électronique réalisée avec le traitement de textes Microsoft Word 2004 pour Macintosh.

Mise en page sur papier format : LETTRE (US letter), 8.5’’ x 11’’)

Édition numérique réalisée le 18 septembre 2007 à Chicoutimi, Ville de Saguenay, province de Québec, Canada.

\section{CFait avec}




\section{Ginette Paquet}

Ministère de la Santé et des Services sociaux ;

\section{"Facteurs sociaux de la santé, de la maladie et de la mort”.}
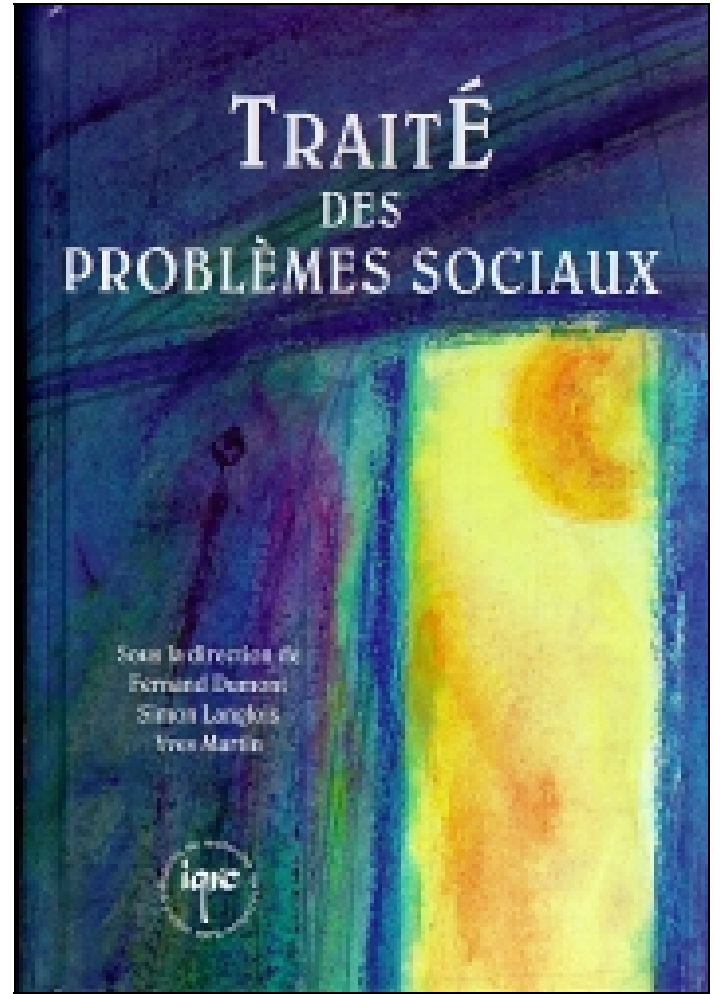

Un article publié dans l'ouvrage sous la direction de in ouvrage sous le direction de Fernand Dumont, Simon Langlois et Yves Martin, Traité des problèmes sociaux. Chapitre 11, pages 223 à 244. Québec: Institut québécois de recherche sur la culture, 1994, 1164 pages. 


\section{Table des matières}

Introduction

1. Les conceptions

1.1. La santé et le bien-être

1.2. L'inégalité d'accès à la santé et au bien-être

2. $\quad$ L'inégalité sociale devant la durée de la vie, la santé et la maladie

2.1. La situation internationale

2.2. La situation canadienne

2.3. La situation québécoise

L'espérance de vie

Les incapacités

La périnatalité

La santé mentale

Les habitudes de vie

Les problèmes d'adaptation et d'intégration sociale

3. Des hypothèses explicatives: l'influence des facteurs sociaux

3.1. La répartition de la richesse ou la pauvreté relative

3.2. L'environnement social et la santé

4. Conclusion

5. Bibliographie sélective

Résumé par Serge Cantin 
Ginette Paquet

Ministère de la Santé et des Services sociaux

"Facteurs sociaux de la santé, de la maladie et de la mort".

Un article publié dans l'ouvrage sous la direction de in ouvrage sous le direction de Fernand Dumont, Simon Langlois et Yves Martin, Traité des problèmes sociaux. Chapitre 11, pages 223 à 244. Québec: Institut québécois de recherche sur la culture, 1994, 1164 pages.

\section{Introduction}

L'accès universel à des soins de santé de qualité constitue l'un des principes fondamentaux de notre société. Nous y consacrons d'ailleurs beaucoup d'argent. Les services de santé représentent au Canada un secteur d'activité économique considérable, l'un des plus importants parmi les pays modernes. Au Québec, ce secteur monopolise à lui seul près de $9 \%$ de la richesse collective (11\% aux États-Unis), c'est-à-dire plus de 10 milliards de dollars, comparativement à $4 \%$ il y a trois décennies. Les services médicaux, les soins hospitaliers, les tests en laboratoire et les médicaments vendus sur ordonnance accaparent la plus grande partie de notre argent et de notre attention lorsqu'il est question des problèmes de santé. L'idée que l'état de santé dépend du nombre de médecins et d'hôpitaux disponibles n'est-elle pas très répandue?

S'il était démontré que le système de soins contribue avantageusement à la richesse collective d'une nation et qu'il constitue le principal déterminant de l'amélioration de la santé de la population, légitime- 
ment, la croissance de ce secteur ne devrait plus susciter de débat. Mais de telles démonstrations n'existent pas ${ }^{1}$. Selon plusieurs économistes:

Des investissements dans les secteurs plus compétitifs de l'économie seraient, disent-ils, préférables, même pour l'amélioration de la santé. Ces secteurs produisent en effet plus de richesse et de bien-être que le secteur des soins, bref des conditions dont l'histoire nous a démontré l'extrême importance pour la santé de la population ${ }^{2}$.

Alors comprendre pourquoi les citoyens de certains pays, qui dépensent moins que nous pour les soins de santé, sont en meilleure santé et aussi pourquoi l'augmentation des sommes consacrées aux services de santé conventionnels dans les pays développés n'a pas forcément d'effet positif sur l'état de santé et de bien-être collectif nous semble essentiel.

Il n'est pas, de toute évidence, dans notre intention de traiter ici de l'ensemble des explications. Notre réflexion sera plus modeste ; nous tenterons de cerner sommairement l'influence contemporaine des facteurs sociaux sur la santé, la maladie et la mort dans les sociétés industrialisées. Dans un premier temps, nous ferons le point sur la définition de quelques concepts, puis nous dresserons un bilan de l'inégalité sociale devant la maladie et la mort afin de faire ressortir le rôle des facteurs sociaux. Enfin, de ce bref tour d'horizon des connaissances disponibles sur la relation entre le social et la santé collective émaneront les avenues prioritaires de recherche.

1 Marc Renaud, «Santé: le sociologue au pays des merveilles», Cahiers de recherche sociologique, 14, printemps 1990, p. 171-179.

2 Ibid., p. 177. 


\section{LES CONCEPTIONS}

\subsection{La santé et le bien-être}

$\underline{\text { Retour à la table des matières }}$

La conception de la santé a évolué dans le temps. On recense à présent de multiples définitions de la santé. Il est de plus en plus question de santé globale, santé fonctionnelle, santé perçue, santé objective, santé positive... La santé et le bien-être sont maintenant perçus comme une ressource de la vie quotidienne et se définissent par « la capacité physique, psychique et sociale d'une personne d'agir dans son milieu et d'accomplir les rôles qu'elle entend assumer, d'une manière acceptable pour elle-même et pour les groupes dont elle fait partie » 3 .

Il n'y a pas si longtemps, toutefois, la santé était définie par l'absence de maladie. Cette conception demeure encore répandue dans bien des milieux. De plus, cette définition traditionnelle implique habituellement la présence de l'équation "santé = soins de santé ». Car depuis plus de cinquante ans, les termes "santé » et " soins de santé » ont été considérés comme synonymes, même si l'accroissement des dépenses du système de santé conventionnel ne produit pas nécessairement les effets correspondants sur l'amélioration générale de la santé de la population. On remarque d'ailleurs que l'opinion publique est fortement monopolisée depuis quelques années par deux éléments. Le premier, le sous-financement des services et des soins de santé déploré par les producteurs de services ainsi que leurs revendications afin d'augmenter les ressources et, le deuxième élément, l'inquiétude des

3 Ministère de la Santé et des Services sociaux, La politique de la santé et du bien-être, Québec, 1992, p. 11. 
gouvernements quant à la part des deniers publics accaparée par les services de santé. Cette perception traditionnelle de la santé escamote, semble-t-il, non seulement les dimensions psychologiques de la santé, mais aussi les causes sociales de la mauvaise santé collective et représente un obstacle à une compréhension plus large du phénomène.

Pour une meilleure compréhension de la santé et du bien-être des populations, l'Institut canadien de recherches avancées (ICRA) propose l'idée fondamentale suivante: «Aujourd'hui la santé collective est fortement liée à la capacité des nations de créer et de distribuer de la richesse - bien plus qu'à la dispensation de soins médicaux ${ }^{4}$.» Compte tenu de l'existence des nombreux autres déterminants de l'état de santé et de bien- être (environnement physique et social, bagage génétique de l'individu, réactions comportementales et immunologiques, importance de la productivité et de la richesse individuelle et nationale), l'ICRA considère le système de soins officiel comme un facteur parmi d'autres. Cet organisme formule par conséquent une mise en garde:

L'accroissement des dépenses de santé risque de devenir un choix moins productif pour l'amélioration de la santé des populations. En effet, une croissance rapide de ces dépenses constituera un fardeau pour les économies modernes; elle réduira leur efficacité et leur compétitivité commerciale et diminuera leur capacité à créer de la richesse 5 .

Avant d'examiner les facteurs explicatifs de l'état de santé et de bien-être collectif, couramment appelés les déterminants, dressons un bilan des connaissances à propos de l'inégalité sociale devant la durée de la vie, la santé et la maladie. Un tel bilan devrait permettre une

4 L'institut canadien de recherches avancées, Les déterminants de la santé, publication $n^{\circ}$ 5, Toronto, septembre 1991, p. 4.

5 Ibid. 
meilleure compréhension des déterminants sociaux de l'état de santé et de bien-être d'une population.

\subsection{L'inégalité d'accès à la santé et au bien-être}

\section{$\underline{\text { Retour à la table des matières }}$}

Lorsqu'il est question d'inégalités d'accès à la santé et au bien-être, on note fréquemment de la confusion. Certains parlent d'inégalités à propos de la qualité et du niveau de santé entre différents groupes de la population alors que d'autres se préoccupent des inégalités quant à la disponibilité et la dispensation des services, et ce, sans toujours opérer de distinction entre les deux ${ }^{6}$. Il est aussi nécessaire de se demander si les écarts observés sont inévitables ou bien s'ils s'avèrent inacceptables et injustes.

Clarifions en premier lieu les écarts de niveaux de santé observés entre différents groupes de la population. La portion de ces écarts attribuable à des variations biologiques doit être considérée comme inévitable et non pas injuste. Certaines différences dans l'état de santé entre plusieurs groupes d'âge peuvent être placées dans cette catégorie. Par exemple, la grande prévalence des maladies coronariennes chez les hommes de 70 ans comparativement à ceux de 20 ans ne peut être attribuée à des injustices, elle est davantage liée au processus de vieillissement chez l'humain. Les plus grands écarts entre différents groupes de la société (incluant les différences entre hommes et femmes) ne peuvent cependant être attribués à des facteurs biologiques, d'autres facteurs sont en cause. Pour savoir si les états de santé observés doivent être considérés comme injustes, il s'agit d'identifier si en majeure partie ils dépendent de situations choisies par les personnes

6 M. Whitehead, «The Concepts and Principles of Equity and Health», Health Promotion International, 6, 3, 1991, p. 217-228. 
ou si les facteurs en cause sont principalement hors de leur contrôle direct. Par exemple, le manque de ressources des personnes défavorisées réduit leurs possibilités de choix. Ces personnes doivent souvent vivre dans des quartiers peu sécuritaires, s'entasser dans des logements, accepter des emplois dangereux et salissants ou vivre de fréquentes expériences de chômage. Les mauvais états de santé provenant de facteurs environnementaux sont clairement injustes. L'injustice s'accroît lorsque les problèmes tendent à se multiplier et à se concentrer, se renforçant les uns les autres, créant ainsi des groupes très vulnérables en matière de santé.

Dans la littérature, en règle générale, on regroupe les facteurs expliquant les écarts de santé et de bien-être en sept grandes catégories ${ }^{7}$ :

- les variations biologique s;

- les comportements nocifs pour la santé choisis librement, tels que la pratique de certains sports ou la participation à certains loisirs ;

- la transition des bénéfices sur la santé d'un groupe par rapport à un autre, quand le premier adopte plus rapidement des comportements sains pour la santé ;

- les effets néfastes sur la santé lorsque les possibilités de choisir des styles de vie sont sévèrement limités ;

- l'exposition à l'insalubrité et à des conditions de vie et de travail difficiles ;

- l'accès inadéquat à des services de santé essentiels et à d'autres services publics ;

- la sélection naturelle ou la relation entre la santé et la mobilité sociale voulant que les personnes malades glissent vers le bas de l'échelle sociale.

7 Ibid., p. 219. 
Un consensus se dégage à propos de ces sept catégories de facteurs: les écarts de santé influencés par les trois premières catégories ne peuvent être classés comme des disparités de santé injustes et évitables, alors que les quatrième, cinquième et sixième catégories de facteurs sont reconnues par plusieurs comme étant évitables et que les disparités de santé qui en résultent peuvent être qualifiées d'injustes. Enfin, la septième catégorie, la tendance des personnes malades à devenir pauvres: le mauvais état de santé peut à l'origine avoir été inévitable mais les bas revenus des personnes malades devraient pouvoir être prévenus et sont considérés comme injustes. Ces sept catégories de facteurs explicatifs des disparités de santé ne sont pas mutuellement exclusives, elles sont liées. Toutefois, le rôle des facteurs biologiques dans la mobilité sociale descendante des personnes malades s'avère minime en comparaison de l'influence des facteurs socioéconomiques et environnementaux, incluant les styles de vie. L'équité relève donc de la création de possibilités égales d'acquérir ou de maintenir la santé entraînant une réduction des écarts de santé et de bienêtre.

Quant à l'égalité d'accès aux services de santé, elle peut être définie de maintes façons. Néanmoins, l'accessibilité égale aux services pour des besoins comparables, l'utilisation égale des services pour des besoins équivalents et la même qualité de soins pour tous et toutes constituent les trois principales dimensions d'une définition opérationnelle 8 .

Certaines caractéristiques individuelles telles que la pauvreté, la race, le sexe, l'âge et la religion peuvent compromettre l'accessibilité égale aux services pour des besoins équivalents. Un accès complet aux soins peut s'avérer très limité pour des usagers à cause de barrières financières, organisationnelles et culturelles. L'accessibilité est

8 H. Leenan, Equality and Equity in Health Care, Paper presented at the WHO $\backslash$ Nuffield Centre for Health Service Studies Meeting, Leeds, 22-26 juillet 1985, cité par M. Whitehead, loc. cit. 
aussi dépendante de la répartition des ressources affectées aux services et aux soins de santé entre les régions urbaines, rurales, prospères ou défavorisées. De plus, si les fonds alloués sont dépensés dans une très large proportion pour des services médicaux de haute technologie, cela peut compromettre l'accessibilité aux autres soins et services.

Pour la deuxième dimension de l'égalité d'accès aux soins, l'utilisation égale des services pour des besoins équivalents, les écarts observés dans l'utilisation des services par différents groupes ne signifient pas nécessairement que ces écarts soient inéquitables. Une petite proportion de ces différences est attribuable aux personnes qui exercent leurs droits de ne pas utiliser les services pour des raisons religieuses ou éthiques. Mais lorsque l'accès aux services est limité par des désavantages sociaux, culturels ou économiques, une discrimination positive peut être légitimement mise de l'avant pour faciliter l'utilisation des services d'immunisation, par exemple, ou celle d'autres services préventifs dans des zones déficientes.

Enfin, la troisième dimension de l'accès équitable aux services, la même qualité de soins pour tous, est primordiale car elle s'avère fragile dans plusieurs sociétés. Explicitement, elle signifie que chaque personne doit avoir une probabilité égale d'être sélectionnée pour l'obtention de services ou de soins requis. La procédure de sélection doit donc reposer sur le besoin plutôt que sur l'influence sociale. Cette probabilité devient plus critique en période de restrictions budgétaires. 


\section{L'INÉGALITÉ SOCIALE DEVANT LA DURÉE DE LA VIE, LA SANTÉ ET LA MALADIE}

\subsection{La situation internationale}

$\underline{\text { Retour à la table des matières }}$

L'association entre un mauvais état de santé et l'appartenance aux couches socio-économiques les moins favorisées est étudiée depuis longtemps. Toutes les sociétés analysées présentent des variations de l'état de santé de la population en fonction de la classe sociale, du revenu, de l'éducation, de la situation professionnelle et de la qualité de l'environnement social. Plusieurs pays industrialisés se préoccupent de la persistance des inégalités sociales par rapport à la santé et au bienêtre. Au bureau régional européen de l'Organisation mondiale de la santé (OMS), la stratégie mondiale de la «Santé pour tous en l'an 2000» a été bâtie autour de cette thématique.

Les États membres de la Région européenne de l'OMS ont évalué la stratégie et les buts choisis pour atteindre "La santé pour tous en l'an 2000 ». Un article récent identifie les principaux progrès accomplis quant aux préalables à la santé et aux problèmes d'équité. À propos des préalables à la santé, les auteurs de l'article signalent:

La paix et la justice sociale, une alimentation appropriée, une éducation adéquate, un revenu suffisant, un rôle utile pour chacun dans la société, un approvisionnement en eau satisfaisant, un logement dé- 
cent, sont les préalables sans lesquels il ne saurait être question de santé, de croissance ou de développement ${ }^{9}$.

La situation se serait améliorée pour certains de ces préalables, mais il y a peu d'indication que les inégalités socio-économiques entre les pays et à l'intérieur des pays soient en voie de diminuer. Dans les pays de la Communauté économique européenne, l'écart entre les régions les plus riches et les plus pauvres, en prenant en compte les différences de coût de la vie, est de l'ordre de 3 à 1 . Ces écarts sont encore plus considérables au sein de certains pays et ils peuvent varier du simple au quadruple entre extrêmes de classe sociale.

Comme il est difficile de comparer les degrés d'inégalité concernant la santé entre les pays, les évaluateurs doivent se limiter aux données sur l'existence d'inégalités à l'intérieur de chaque État membre. Des différences considérables persistent pour la mortalité ou l'espérance de vie, au désavantage, soit des zones urbaines industrialisées (Norvège, Pologne, Royaume-Uni, Tchécoslovaquie), soit des zones rurales (Grèce, Hongrie, Yougoslavie). De plus, les taux de mortalité infantile varient de 7,2 à 12,4 pour 1000 naissances vivantes en Norvège, par exemple, et de 13,6 à 28,6 en Pologne. Des études longitudinales ont mis en évidence des différences persistantes de mortalité en France et au Royaume-Uni entre les catégories les plus privilégiées et les moins favorisées de la société. Le Danemark, la Finlande, la Hongrie, la Norvège et la Suède ont aussi indiqué des écarts dans les taux de mortalité entre catégories professionnelles et en fonction du niveau d'éducation ou du revenu. Les plus grandes disparités liées aux causes socio-économiques s'observent pour la mortalité des hommes d'âge adulte. Pour la mortalité infantile, des écarts considérables sont

9 S.E. Curtis, A. Taket, R Prokhorskas, M.A. Shabanah et M.C. Thuriaux, «Vers la santé pour tous dans la région européenne de l'OMS. Surveillance des progrès accomplis. II; Conditions préalables: bilan démographique et sanitaire», Épidémiologie et santé publique, 37, 1989, p. 299. 
notés entre groupes de niveaux d'instruction différents, par exemple au Portugal, en Suède, en Yougoslavie et au Royaume-Uni 10.

Les sous-titres du chapitre XII du livre Mortalité et causes de décès en France 11 illustrent avec éloquence l'inégalité sociale devant la mort rencontrée en France. En voici un aperçu:

Du professeur au manoeuvre: une mortalité croissante; Le classement des catégories sociales est le même pour la mortalité infantile que pour celle des adultes; La catégorie socio-professionnelle du mari pèse sur la mortalité de son épouse; L'influence du milieu social se prolonge aux âges élevés pour les hommes comme pour les femmes; Les différences de mortalité sont plus marquées dans les agglomérations; L'inégalité s'est légèrement accrue en vingt ans (de 1960 à 1980); Les personnes sans activité ont une mortalité plus élevée que les actifs du même âge; La vie de famille protège; La propriété sélectionne.

Quant à la morbidité, un énorme travail reste à accomplir avant de pouvoir obtenir des données comparables sur le plan international. Toutefois, plusieurs pays disposent de données nationales intéressantes. La Suède a démontré que les mères célibataires peu scolarisées donnent plus fréquemment naissance à des bébés de poids insuffisant (moins de 2,5 kilos). Au Royaume-Uni, la prévalence du poids insuffisant à la naissance est plus élevée dans les catégories sociales défavorisées. Dans ce dernier cas, le gradient semble même avoir augmen-

10 P. Aiach, R Carr-Hill S. Curtis et R. Illsley, Les inégalités sociales de santé en France et Grande-Bretagne. Analyse et étude comparative, Paris, INSERM, La Documentation française, 1988, cité par S.E. Curtis, A. Taket, R Prokhorskas, M.A. Shabanah et M.C. Thuriaux, loc. cit.; Infant and Perinatal Mortality 1986 by Birthweight, Londres, Office of Population Censuses and Surveys, 1988 (DH3 88/1), cité par S.E. Curtis, A. Taket, R Prokhorskas, M.A. Shabanah et M.C. Thuriaux, loc. cit.

11 G. Desplanques, «L'inégalité sociale devant la mort», Mortalité et causes de décès en France, Collection «Grandes enquêtes en santé publique et épidémiologie», INSERM, France, 1990, chapitre Xll, p. 257-287. 
té. À l'échelle européenne, la tuberculose demeure plus fréquente chez les immigrants et les classes défavorisées et sa persistance est considérée comme un signe important d'inégalité. La prévalence de la carie dentaire est, elle aussi, liée à la classe sociale, et ce sont les plus riches qui présentent l'amélioration la plus rapide ${ }^{12}$. D'autres données montrent l'existence dans plusieurs pays de gradients liés au statut socioéconomique ; au Royaume-Uni entre autres, le poids adulte, la tension artérielle, la fonction respiratoire, la taille présentent ce gradient. Ces inégalités sont surtout marquées chez les enfants et chez les adultes de plus de 25 ans. Les chercheurs insistent sur ce dernier constat, car la réapparition des inégalités après l'âge de 25 ans a des implications importantes pour le problème de l'équité en matière de santé ${ }^{13}$.

Des inégalités quant à l'accessibilité aux services prévalent à l'intérieur des pays selon les données disponibles. Voici quelques exemples d'inégalités rapportés par des pays européens : l'Italie et la Pologne (distribution des services ou du personnel de santé), la Yougoslavie (consommation de soins périnatals), et la Finlande (répartition des médecins généralistes dans les zones urbaines) ${ }^{14}$. On rapporte par ailleurs dans plusieurs pays l'implantation de mesures en vue de réduire ces disparités. Ces efforts visent à axer les ressources sur les groupes les plus exposés aux risques à l'égard de la santé et du bienêtre, à adapter les services pour les rendre plus accessibles à ces groupes, ou à élaborer une politique nationale en vue de réduire les inégalités (par exemple, un ensemble minimal de prestations sociales spécifiques axées sur les zones défavorisées). On remarque que dans la

12 M. Hobdell et G. Gavin, «Dental Caries and Periodontal Disease?», dans: A.J. Silman et S.P.A. Allwright (sous la direction de), Elimination or Reduction of Diseases? Opportunities for Health Service Action in Europe, Oxford, Oxford Medical Publications, 1988, p. 140-156, cité par 8.E. Curtis, A. Taket, R. Prokhorskas M.A. Shabanah et M.C. Thuriaux, loc. cit.

13 S.E. Curtis, A. Taket, R. Prokhorskas, M.A. Shabanah et M.C. Thuriaux, loc. cit., p. 300.

14 Ibid. 
prestation de soins de santé préventifs pour les jeunes, plusieurs pays renforcent les activités visant les groupes à risques élevés.

D'autre part, dans un numéro de Social Science and Medicine intitulé «Special Issue: Health Inequities in Europe 15 », des chercheurs font le point sur l'état des connaissances. L'indice de défavorisation socio-économique étant différent d'un pays à l'autre, ils concluent, eux aussi, qu'il est très difficile, pour ne pas dire impossible, d'établir des comparaisons entre les pays, pour différentes causes de mortalité ou diverses maladies, selon le statut socio-économique. En dépit de cette constatation, la réflexion de plusieurs groupes de chercheurs apporte un éclairage intéressant.

On y apprend que la République fédérale allemande observe une diminution des inégalités au chapitre de la santé entre les cols bleus et les cols blancs. Cependant, les auteurs de l'article notent un accroissement des inégalités entre les chômeurs et l'ensemble des travailleurs. Les Finlandais soutiennent pour leur part que l'influence du statut socio-économique sur la santé et la mortalité est plus forte et plus fréquente dans les sociétés industrialisées, comparativement aux pays en développement. Quant à la République d'Irlande, les chercheurs constatent que les plus fortes inégalités sociales de mortalité y sont liées aux maladies cardiovasculaires, aux maladies respiratoires et au cancer. Les chômeurs de longue durée constituent le groupe le plus vulnérable. En Israël, on évalue à $94,5 \%$ la population qui bénéficie d'un système de soins assuré par l'État. Néanmoins, les inégalités de santé et d'accès aux services persistent pour certaines populations. Deux types de causes sont identifiées. D'une part, on pointe la scolarité, l'occupation, le revenu, les valeurs culturelles, la motivation et les comportements sains. D'autre part, les Israéliens retiennent les facteurs reliés à la structure du système de santé. Les chercheurs italiens insistent quant à eux sur l'association qui prévaut entre les variables

15 Peter J.M. McEvan (sous la direction de), «Health Inequities in Europe», numéro spécial de Social Science and Medicine, 31, 3, 1990, p. 221-420. 
socio-économiques, l'état de santé et la façon d'utiliser les services. Pour les Pays-Bas, l'intérêt se porte vers une «constellation de facteurs de risques»: l'occupation, le degré d'instruction, l'expérience d'événements stressants, le réseau social limité, la consommation d'alcool et le tabagisme régulier ainsi que l'alimentation riche en gras. Quant aux Norvégiens, ils cherchent à comprendre pourquoi certains membres d'un groupe social sont exposés à des problèmes de santé et d'autres pas. Ils constatent de plus que certaines campagnes de prévention, en ne ciblant pas adéquatement les groupes défavorisés sur le plan socioéconomique, ont eu peu d'effet et qu'elles ont même contribué à augmenter les inégalités. Tout en reconnaissant que les déterminants de la santé sont complexes et variés, les chercheurs espagnols démontrent que la pauvreté, les conditions de vie, le revenu, et le statut socioprofessionnel expliquent une part considérable de la variation.

Dans presque tous les pays développés, on observe des inégalités marquées de l'état de santé des populations qui se mesurent en termes d'espérance de vie, de mortalité, d'incapacité, d'incidence ou de prévalence de maladies spécifiques. Ces écarts de santé sont liés à des facteurs sociaux, économiques et culturels, puisque dans la plupart des sociétés modernes, l'instauration de systèmes de santé publics a facilité l'accès aux services de santé ainsi que leur utilisation. Comme par le passé, les personnes riches vivent encore plus longtemps et en meilleure santé que les personnes pauvres ${ }^{16}$. Même si l'évolution technologique nous a procuré une meilleure capacité technique dans la prévention et le traitement des maladies, des différences importantes de mortalité et de morbidité demeurent entre les classes sociales. Ces écarts sont particulièrement prononcés pendant la période périnatale et à l'âge adulte entre 35-55 ans ${ }^{17}$.

16 G. Davey Smith, M. Bartley et D. Blane, «The Black Report on Socioeconomic Inequalities on Health Ten Years On», British Medical Journal, 1990, 301, p. 373-377.

17 J.S. House et al., «Age, Socioeconomic Status, and Health», Milbank Quarterly, 68, 3,1990, p. 384-411. 


\subsection{La situation canadienne}

$\underline{\text { Retour à la table des matières }}$

Des recherches effectuées au Canada démontrent l'existence de ce gradient entre classes sociales. L'étude réalisée auprès de plus de 500 000 hommes canadiens ayant pris leur retraite depuis l'entrée en vigueur du Régime des pensions du Canada indique un gradient indéniable selon le revenu. 18 Ainsi, pendant les douze années qui précèdent la retraite, plus le revenu d'un homme est élevé, plus ses chances de vivre longtemps sont grandes. Les hommes qui se situent parmi les $5 \%$ ayant le revenu le plus bas, avant la prise de la retraite, présentent deux fois plus de risques de mourir entre 65 et 70 ans que les 5\% ayant les plus hauts revenus. Deux autres chercheurs, Wilkins et Adams, ont démontré que la population canadienne comporte un gradient très étendu de mortalité et de morbidité entre les niveaux socioéconomiques lorsque l'on procède à une analyse par division de recensement plutôt que par individu ${ }^{19}$. Ces études proposent que même s'ils meurent plus jeunes, les Canadiens pauvres ne présentent pas moins de maladies et moins d'incapacités dans les dernières années de leur vie. Au contraire, les personnes plus riches peuvent espérer jouir en moyenne de 12 années de vie en bonne santé de plus que les personnes pauvres.

18 M. Wolfson et al., "Career Earnings and Death, A Longitudinal Analysis of Older Canadian Men», Canadian Institute for Advanced Research, Population health working paper 12, Toronto, février 1991.

19 Russell Wilkins et O. Adams, Healthfullness of Life, Institute for Research on Public Policy, Montréal, 1978. 


\subsection{La situation québécoise}

$\underline{\text { Retour à la table des matières }}$

Une constante se dégage de plusieurs travaux québécois, dont ceux de la Commission d'enquête sur les services de santé et les services sociaux et de l'enquête Santé Québec : celle de la persistance d'écarts importants en matière de santé et de bien-être entre les mieux nantis et les plus démunis. Dans l'ensemble, certes, la situation socio-sanitaire des Québécoises et des Québécois s'est améliorée. Toutes et tous n'ont cependant pas profité de ces gains. Le statut socio-économique s'avère toujours un déterminant majeur de l'état de santé et de bien-être de la population. La persistance et l'importance de la relation entre le niveau socio-économique et l'état de santé révèlent, dans une certaine mesure, l'échec de nos politiques et programmes pourtant pavés de bonnes intentions.

Que l'on considère l'espérance de vie en bonne santé, la mortalité, les principales maladies ou les difficultés d'adaptation ou d'intégration sociale, ou encore l'adoption de saines habitudes de vie, les Québécois et les Québécoises défavorisés font piètre figure comparativement aux mieux nantis.

\section{L'espérance de vie}

L'espérance de vie suit très fidèlement les courbes du revenu, de la scolarité et de la profession. Par exemple, en dépit d'une baisse importante des indices de mortalité dans la région de Montréal, on constate encore dans les années 1990 des disparités importantes. Un écart de 10 ans est observé entre les espérances de vie des populations de quartiers favorisés et défavorisés à Montréal. C'est dans la partie centresud de Montréal que l'on retrouve à la fois les niveaux de mortalité les 
plus élevés et les plus fortes proportions de personnes pauvres. Les résidents d'Outremont/Mont-Royal présentent une longévité moyenne de 81,5 ans, comparativement à 69,8 ans pour ceux de Pointe-SaintCharles 20.

Pour l'espérance de vie en bonne santé, qui tient compte de la durée et de la qualité de vie, l'écart entre riches et pauvres atteindrait 14,4 ans ${ }^{21}$. De plus, les écarts selon le revenu augmentent pour deux causes de mortalité: le suicide et le cancer du poumon. Les personnes pauvres sont celles chez qui l'on a observé, au cours des dernières années, une augmentation importante du nombre de décès par suicide ou par cancer du poumon ${ }^{22}$. L'écart de la mortalité selon le revenu dans les régions urbaines du Canada semble toutefois avoir diminué un peu de 1971 à 1986.

D'autres travaux ont révélé d'importantes disparités régionales tant dans les déterminants de l'état de santé (pour diverses pathologies) ou encore dans les conséquences de cet état de santé (incapacité, recours aux services, médication) ${ }^{23}$. Ces travaux permettent de mettre en évidence les populations les plus vulnérables au Québec : les résidents des vieux centres-villes et de certains secteurs limitrophes d'agglomérations et villes, des capitales régionales et de la métropole ; et les résidents de l'arrière-pays et de certaines petites villes de la périphérie.

20 Robert Choinière, «Les disparités géographiques de la mortalité dans le Montréal métropolitain, 19841988: études écologiques des liens avec les conditions économiques, sociales et culturelles», Cahiers québécois de démographie, 20,1,1991, p. 117-146.

21 Russell Wilkins, «Health Expectancy by Local Area in Montréal: A Summary of Findings», Canadian Journal of Public Health, 77, mai-juin 1986, p. 216220.

22 R. Wilkins, O. Adams et A. Brancker, «Évolution de la mortalité selon le revenu dans les régions urbaines du Canada entre 1971 et 1986», Rapports sur la santé, Statistique Canada, mars 1990, p. 137-174.

23 R. Pampalon, G. Raymond, D. Beaudry et D. Gauthier, La santé à la carte. Une exploration géographique de l'enquête Santé Québec, Québec, Les Publications du Québec, 1990, 60 p. 


\section{Les incapacités}

L'enquête Santé Québec a révélé que les Québécois défavorisés âgés de 45 à 64 ans resemblent physiquement aux Québécois mieux nantis de 65 ans et plus ${ }^{24}$. Les incapacités sévères varient selon le groupe social: en fait, $14,8 \%$ des personnes très défavorisées présenteraient des incapacités sévères, contre $1,0 \%$ en milieu favorisé. Plus de 21\% des 45-64 ans vivant en milieu défavorisé et très défavorisé souffriraient d'une incapacité sévère alors qu'on en retrouve seulement $15 \%$ et $9 \%$ chez les 65 ans et plus des milieux moyens et favorisés. Les mêmes écarts s'observent pour les incapacités et les symptômes de troubles mentaux.

\section{La périnatalité}

Le taux de mortalité infantile est deux fois plus élevé en milieu défavorisé 25 . Les enfants y sont plus souvent victimes d'accidents et de maladies infectieuses ou respiratoires. Dans ces milieux, 12\% des bébés qui naissent sont de poids insuffisant (moins de 2,5 kilos), deux fois plus que dans les milieux favorisés. Certains quartiers pauvres du Québec présentent des taux de naissance de bébés de poids insuffisant comparables à ceux de pays en développement.

\section{La santé mentale}

Le stress engendré par la pauvreté a également d'importantes répercussions sur la santé mentale. L'enquête Santé Québec a montré qu'il y a environ deux fois plus de personnes défavorisées que de personnes favorisées qui présentent un niveau élevé de détresse psycho-

24 C. Colin, J.-P. Lavoie et C. Poulin, Les personnes défavorisées, Et la santé, ça va?, Québec, Les Publications du Québec, 1989,119 p.

25 C. Colin, H. Desrosiers et al., Naître égaux et en santé, ministère de la Santé et des Services sociaux, Québec, 1989, 153 p. 
logique: 30\% contre 16,2\%. Même si l'importance des événements stressants varie surtout en fonction de l'âge, des écarts importants existent entre les différents groupes socio-économiques. Ainsi, 37\% des personnes très défavorisées ont connu un niveau de stress moyen ou élevé durant la dernière année précédant l'enquête. Cette proportion est de $35 \%$ chez les groupes défavorisés et tombe à $24 \%$ chez les moyens et à $16 \%$ chez les favorisés. De plus, les personnes très défavorisées ont près de trois fois plus d'idées suicidaires et commettent aussi trois plus fréquemment que les autres des tentatives de suicide au cours de leur vie. Il n'est donc pas étonnant de constater que les décès par suicide sont plus fréquents en milieu défavorisé et même que l'écart entre les groupes socio-économiques augmente ${ }^{26}$.

Les scores obtenus aux indices de détresse psychologique et de santé mentale positive de l'enquête Santé Québec montrent que les parents de familles monoparentales, femmes et hommes, éprouvent un niveau de détresse plus élevé et démontrent un niveau de santé mentale positive plus faible que leurs homologues de familles biparentales. Les données sur les idées suicidaires vont dans le même sens 27.

\section{Les habitudes de vie}

Quant aux habitudes de vie, l'indice de l'enquête Santé Québec qui tient compte de la consommation de tabac et d'alcool, de la pratique d'exercice physique, du sommeil, du poids en fonction de la taille montre que les personnes très défavorisées présentent la plus grande proportion de mauvaises habitudes de vie : une personne sur quatre. Cette proportion est d'une personne sur cinq pour les personnes défavorisées. Les personnes défavorisées fument plus: 46,3\% de fumeurs réguliers chez les très défavorisées contre $23,7 \%$ pour les personnes

26 R Wilkins, O. Adams et A.M. Brancker, loc. cit.

27 Carmen Bellerose, Élizabeth Cadieux et Ellen Johnson, Les familles monoparentales, Et la santé ça va?, Québec, Les Publications du Québec, 1989, p. 2123. 
favorisées. On retrouve des pourcentages de personnes obèses plus élevés chez les groupes défavorisés et très défavorisés (14\%), comparativement à $9 \%$ et $6 \%$ chez les groupes moyens et favorisés, respectivement.

\section{Les problèmes d'adaptation} et d'intégration sociale

La relation entre la pauvreté et l'incidence des problèmes sociaux a aussi été maintes fois démontrée. On a par exemple constaté que, dans son ensemble, la clientèle des centres de services sociaux est habituellement issue des couches défavorisées de la population ${ }^{28}$. En 1988, alors qu'environ 13\% des familles du Québec vivaient sous le seuil de la pauvreté, c'était le cas de plus de $40 \%$ de l'ensemble des bénéficiaires du Centre de services sociaux du Montréal métropolitain, et probablement des autres centres de services sociaux du Québec.

Même si bien des parents de milieux défavorisés s'avèrent compétents, affectueux et très dévoués, les familles dont les enfants sont placés en famille d'accueil sont, dans plus de $60 \%$ des cas, sous le seuil de la pauvreté. De la même façon, les mauvais traitements physiques infligés aux enfants ont plus de risques de survenir en milieu défavorisé, comme le démontrent les statistiques québécoises et montréalaises. Quant aux enfants négligés, la majorité vivraient à l'intérieur de familles pauvres, monoparentales. En plus de vivre les conséquences de la pauvreté, ces enfants négligés courent un risque important de connaître l'isolement social, le manque d'affection, de stimulation et d'encadrement. Plus tard, l'absentéisme scolaire, la sous- scolarisation ainsi que la délinquance ont de fortes probabilités d'être le lot de ces jeunes 29.

28 Micheline Mayer-Renaud, «Le statut socio-économique: construction d'un indice utile pour la gestion sociale», Sociologie et sociétés, XX, 1, avril 1988, p. 47-59.

29 Micheline Mayer-Renaud, Les enfants du silence. Une revue de la littérature, Thème: négligence associée à la pauvreté, CSSSMM, 1985, 25 p. 
Par contre, la prévalence des cas d'enfants victimes d'abus sexuels ne semble pas varier selon le niveau socio-économique. La violence conjugale ne serait pas non plus le lot des milieux défavorisés, même si les femmes de ces milieux recourent davantage (88\%) aux centres d'hébergement pour femmes violentées ${ }^{30}$. Quant aux personnes âgées, celles qui requièrent des services sociaux s'avèrent parmi les plus défavorisées de leur groupe.

En somme, les Québécoises et les Québécois à faible niveau d'instruction et de revenu présentent plus de problèmes de santé et de problèmes sociaux, et plus de décès prématurés que les autres. Elles courent des risques de mort prématurée, de maladie et d'invalidité supérieurs à la moyenne.

Cette superposition de problèmes engendre évidemment une consommation singulière de services sociaux et de santé chez les clientèles défavorisées; en voici un bref aperçu :

- Une consommation plus élevée de médicaments prescrits, notamment les tranquillisants (72\% contre $51 \%$ chez les personnes favorisées).

- La population défavorisée aurait recours plus souvent à la clinique hospitalière.

- En milieu défavorisé, le médecin généraliste est plus fréquenté que le spécialiste.

- Une fréquence des hospitalisations plus élevée en milieu défavorisé: $13 \%$ contre $7 \%$.

- Les personnes défavorisées consultent trois fois moins souvent le dentiste que les nanties, 6,3\% comparativement à 18,7\%.

30 Ministère de la Santé et des Services sociaux, État de situation sur les revenus des femmes utilisatrices des maisons d'hébergement Z990, dossier du Régime d'assurance publique du Canada, Service de la négociation et de la coordination inter-gouvernementale, Québec, 1990. 
- Une surreprésentation des personnes pauvres parmi la clientèle des centres de services sociaux.

- Le placement d'enfants en famille d'accueil plus fréquent (60\% des cas viennent de familles sous le seuil de la pauvreté).

- La très grande majorité des femmes (88\%) qui fréquentent les maisons d'hébergement pour femmes victimes de violence conjugale ont un revenu personnel inférieur à 12 000\$ par année.

- Une sous-utilisation des services de prévention par les clientèles de milieux défavorisés ${ }^{31}$.

\section{DES HYPOTHÈSES EXPLICATIVES: L'INFLUENCE DES FACTEURS SOCIAUX}

$\underline{\text { Retour à la table des matières }}$

Même si les principales causes de maladie ont beaucoup changé, les écarts de mortalité et de morbidité entre les classes socioéconomiques paraissent assez stables sur une longue période de temps. Par conséquent, cela suppose que des facteurs sous-jacents influencent la prédisposition aux maladies et agissent durant des décennies et même au fil de plusieurs générations. Des facteurs de risque généraux sont donc en cause plutôt que des facteurs de risque individuels 32 .

En 1948, l'identification de ces inégalités de santé et l'espoir que des soins de santé universels parviennent à les réduire ont donné lieu à

31 G. Paquet, Santé et inégalités sociales. Un problème de distance culturelle, Québec, Institut québécois de recherche sur la culture, 1989,131 p.

32 C. Hertzman, J. Frank et R. Evans, «Heterogeneities in Health Status», Canadian Institute for Advanced Research, internal document 3C, Toronto, décembre 1990 . 
la création du National Health Service (NHS) au Royaume-Uni. Mais la Royal Commission on Health Services concluait, dans les années 1970, que malgré l'efficacité du NHS à abolir les barrières financières d'accès aux soins, les écarts de santé selon la classe sociale n'ont pas cessé de s'accroître ${ }^{33}$. À la suite de cette constatation, l'on confia à un comité présidé par sir Douglas Black le mandat d'expliquer les causes de cet échec. Le rapport Black, Inequalities in Health ${ }^{34}$, identifia l'influence considérable des facteurs socio- économiques sur la santé en tant que principale explication.

Puisque l'Angleterre a connu un ralentissement économique au cours de ce siècle, on peut légitimement se poser la question suivante: quelle est la part de l'augmentation de l'écart du gradient de l'état de santé dans ce pays que l'on peut attribuer à cette faible croissance économique ? Mentionnons qu'au cours de cette période, la Suède devenait une nation prospère au sein de laquelle les écarts de revenus s'amenuisaient. De plus, le taux de mortalité pour la classe sociale la moins favorisée est plus bas en Suède que celui de la classe supérieure au Royaume-Uni ${ }^{35}$.

L'Institut canadien de recherches avancées affirme: «Dans toutes les nations, la mortalité générale et la plupart des formes de morbidité (quand elles sont bien mesurées) suivent un gradient entre les classes socio-économiques. Un faible revenu ou un bas niveau socioéconomique sont associés à une mauvaise santé ${ }^{36}$.» L'ICRA insiste sur le fait que cette relation n'est pas uniquement attribuable à une extrême privation au bas de l'échelle.

33 Great-Britain Royal Commission on National Health Service, Report of the Royal Commission on the National Health Service (The Merrison Report), London, HMSO, 1979.

34 D. Black, J. Morris, C. Smith et P. Townsend, Inequalities in Health : The Black Report, New York, Penguin Books, 1982.

35 D. Vagero et O. Lundberg, «Health Inequalities in Britain and Sweden», Lancet, ler juillet 1989, p. 35-36.

36 L’Institut canadien de recherches avancées, op. cit., p. 7. 
On observe que le gradient de santé entre les classes sociales est continu et ne présente pas de point de rupture. Les personnes qui appartiennent aux couches sociales supérieures semblent en meilleure santé que celles qui se trouvent à l'échelon suivant, même si ces dernières sont placées plus haut que le restant de la population en termes de revenu et rang social; ou quel que soit le facteur déterminant.

En ce sens, l'étude longitudinale Whitehall, dirigée par Michael Marmot et réalisée à Londres auprès de la fonction publique ${ }^{37}$, révèle la présence d'une variation importante de l'état de santé et de la mortalité selon l'importance du poste occupé dans l'organisation. Cette variation s'observe même si les fonctionnaires occupant des postes situés au bas de la hiérarchie possèdent des revenus assez importants comparativement à la plupart de leurs concitoyens ou à leurs ancêtres. De telles différences entre les échelons les plus élevés et les moins élevés et aussi celles observées entre les plus élevés et ceux qui les suivent immédiatement sont fondamentales pour comprendre les facteurs déterminants. Ces différences se rencontrent pour la plupart des causes de mortalité. Elles semblent influencer les mécanismes de défense des individus contre plusieurs maladies (cancer, maladies coronariennes, infarctus, etc.). Il est aussi fort probable que des interventions destinées à traiter des maladies spécifiques ne réussissent pas à atteindre l'origine des problèmes et s'avèrent peu efficaces. Car même si l'on parvient à traiter efficacement une maladie précise, une autre souvent lui succède.

37 M.G. Marmot et T. Theorell, «Social Class and Cardiovascular Disease: The Contribution of Work», International Journal of Health Sciences, 18, 4, 1988, p. 659-674; M.G Marmot, M.A. Kogevinas et M. Elston, «Social/Economic Status and Disease», Annual Review of Public Health, 8, 1987, p. 11-35. 


\subsection{La répartition de la richesse ou la pauvreté relative}

Retour à la table des matières

On reconnaît assez aisément l'existence de corrélations entre le produit national brut (PNB) per capita et les mesures de l'état de santé comme l'espérance de vie. Mais l'on doit s'intéresser aussi à l'existence de fortes corrélations entre le degré d'équité dans la distribution du produit national et l'état de santé 38 . À ce sujet, l'expérience de la Suède montre qu'il est possible de diminuer les écarts de santé entre les groupes sociaux. Quant à l'exemple du Japon, il illustre très bien les liens entre la prospérité et la santé certes, mais surtout il renseigne sur le rôle de la répartition de la richesse.

Au Japon, l'espérance de vie à la naissance est passée, entre 1955 et 1986, de 63,6 à 75,2 ans pour les hommes, et de 67,8 à 80,9 ans pour les femmes 39 . Afin de saisir l'ampleur du changement, soulignons qu'une telle amélioration supposerait, au Royaume-Uni notamment, l'élimination de tous les décès attribuables aux maladies cardiovasculaires et à la plupart des cancers. La performance économique de la société japonaise est étroitement associée à ses gains de santé remarquables. La part du produit national brut que le Japon consacre au système de santé est l'une des plus basses parmi les pays développés. En 1987, elle correspondait à 6,8\% du PNB, alors qu'au Canada elle était de 8,6\% et aux États-Unis de 11,2\%. Comment expliquer l'augmentation remarquable de l'espérance de vie ? L'explication se trouve probablement du côté de la croissance du PNB du Japon, qui est la

38 R.G. Wilkinson, «Income Distribution and Life Expectancy», British Medical Journal, 304, janvier 1992, p. 165-168.

39 M.G. Marmot et G.D. Smith, «Why Are the Japanese Living Longer?», British Medical Journal, 299, 23-30 décembre 1989, p. 1547-1551. 
plus rapide de tous les pays de l'OCDE, et aussi dans le fait que l'écart relatif de revenu entre les plus pauvres et les $20 \%$ plus riches de la population est le plus faible de tous les pays de l'OCDE. En d'autres mots, parmi les sociétés modernes, le Japon s'avère non seulement le pays qui a connu au cours des dernières décennies la plus rapide croissance économique mais aussi celui où les écarts dans la répartition des revenus entre riches et pauvres sont les plus faibles.

Certains pays peu industrialisés ont réussi à maintenir leur population en meilleure santé que d'autres qui ont pourtant la même richesse per capita ${ }^{40}$. Le Costa Rica, le Sri Lanka et Kerela, en Inde, ont tous un taux de mortalité infantile de 64 par 1000 naissances vivantes et une espérance de vie à la naissance de 61 ans, alors qu'avec le même niveau de revenu per capita, le Pakistan, l'Afghanistan et le Maroc ont des taux de mortalité à la naissance de 173 par 1000 naissances vivantes et que l'espérance de vie moyenne y est de 45 ans. L'état des connaissances nous révèle en outre que les pays où la population est en meilleure santé sont ceux où la culture et la société accordent une place importante aux femmes et à leur scolarisation.

À propos de la répartition de la richesse collective, rappelons que le Rapport mondial sur le développement humain, publié en 1992 par les Nations Unies, classait le Canada au premier rang parmi les pays industrialisés qui détiennent le meilleur indicateur de développement humain. Cet indicateur se compose du revenu national, de l'espérance de vie et du niveau d'instruction. Par contre, toujours selon ce même rapport, le Canada rétrograde de la première à la huitième place lorsque l'indicateur en question est ajusté selon les disparités entre les sexes. De plus, lorsque l'indicateur est ajusté en fonction de la répartition des revenus, le Canada glisse au sixième rang. Donc, même si le Canada est l'un des pays qui offrent les meilleures conditions de vie,

40 J.C. Caldwell, «Routes to Low Mortality in Poor Countries», Population and Development Review, 12, 2, juin 1986, p. 171-220. 
de nombreux Canadiens et Canadiennes ne profitent pas des progrès du développement humain.

Quant à la situation générale du Québec, elle s'est dégradée par rapport aux autres régions canadiennes. Le Québec occupe maintenant le dernier rang pour l'indice global de pauvreté ainsi que pour l'indice de pauvreté des personnes seules, il est au huitième rang pour la pauvreté des familles. La situation des enfants québécois vivant dans des familles monoparentales dirigées par une femme s'est aussi détériorée (41\% des familles monoparentales sont pauvres). Au Québec, comme dans les autres pays industrialisés, l'insuffisance de revenu est relative, puisqu'elle est définie par rapport au niveau de bien-être d'un milieu social donné. Être pauvre signifie une insuffisance d'accès à des biens et à des services de base ainsi qu'à des possibilités qui sont communément offertes aux groupes plus favorisés de la population.

Les privations financières ont des effets indirects et directs sur la santé et le bien-être. Nous avons vu précédemment de nombreuses illustrations de la superposition de problèmes sociaux et de santé découlant de la vie en milieu défavorisé. L'important stress qu'engendre la pauvreté est responsable, en bonne partie, du peu de succès des mesures visant à corriger des habitudes néfastes, tel le tabagisme. L'insuffisance de revenu influence certains choix qui affectent la santé. Par exemple, plusieurs familles à faible revenu doivent consacrer plus de $30 \%$ au logement. Elles sont alors très souvent dans l'obligation de couper sur l'alimentation et les loisirs. Le coût du logement apparaît capital pour l'organisation familiale. Si l'alimentation, l'habillement et les loisirs subissent continuellement des coupures radicales à cause du logement, le stress engendré affectera la santé physique et mentale. Habiter un logement, c'est aussi vivre dans un milieu social donné. On a observé qu'à pauvreté égale, un quartier où les liens sociaux sont encore très vivants présente moins de problèmes de santé et moins de difficultés d'adaptation sociale. 
Les liens étroits entre la scolarité et la santé de même qu'avec les problèmes d'adaptation et de dépendance sociale sont bien connus. On sait par exemple que les gens plus scolarisés ont davantage accès aux services, peuvent plus facilement faire valoir leurs droits et sont plus autonomes. Une scolarité avancée va habituellement de pair avec un bon revenu, un emploi valorisant et l'accès aux loisirs et à la culture. À revenu égal, les gens scolarisés présentent en moyenne un meilleur état de santé et de bien-être. En ce qui a trait à la scolarisation, même si elle s'améliore, la situation du Québec présente plusieurs lacunes. En 1986, près du quart de la population québécoise n'avait pas complété une neuvième année. Dans certains quartiers montréalais, la proportion atteignait 50\%. Environ 10\% des gens étaient analphabètes et 18\% analphabètes fonctionnels. Le Québec se classe à l'avant-dernier rang des provinces canadiennes, juste avant Terre-Neuve, pour l'ampleur de l'analphabétisme. La proportion des jeunes qui ont abandonné l'école secondaire sans avoir obtenu un diplôme s'est maintenue depuis trois ans à 35\%.

D'autre part, plusieurs travaux de recherche ${ }^{41}$, nous ont appris que les attitudes et les perceptions des gestionnaires et des dispensateurs de services s'avèrent des éléments déterminants du succès ou de l'échec des interventions développées à l'intention de la population défavorisée. Les façons de planifier les services et d'intervenir sont le reflet de valeurs culturelles et de styles de vie propres aux classes moyennes ou supérieures. De sorte que la plupart des programmes et des services développés sont loin des préoccupations, des valeurs et du style de vie des milieux populaires et, par conséquent, ils ont peu d'impact auprès de cette clientèle.

41 G. Paquet, op. cit.; D. Mechanic et al., Pathways to Health: The Role of Social Factors, Menlo Park, Kaiser Foundation, 1989, p. 3-75. 


\subsection{L'environnement social et la santé}

$\underline{\text { Retour à la table des matières }}$

Les relations sociales, ou leur absence, s'avèrent un risque majeur ou un facteur de protection important pour la santé et le bien-être d'un individu, selon plusieurs recherches menées aux États-Unis. Une étude californienne révèle que plus les contacts sociaux sont nombreux, plus le taux de mortalité est bas ${ }^{42}$. Ainsi, pour tous les groupes d'âge, cette étude a révélé que les hommes ayant moins de relations sociales risquaient au moins deux fois plus de mourir que les hommes du même âge entretenant plus de relations sociales. Chez les femmes, celles présentant le moins de contacts sociaux étaient trois fois plus à risque de mourir. Avant cette étude californienne, seule la longévité des gens mariés était reconnue ainsi que la relation entre le veuvage et un taux de mortalité plus élevé.

D'autres recherches réalisées aux États-Unis et ailleurs confirment l'existence de «l'association entre toutes les causes de mortalité et différents aspects des relations interpersonnelles, plus spécifiquement le manque de soutien émotionnel, le manque d'activités sociales et le fait de vivre seul. L'association subsiste même après avoir contrôlé pour les variables confondantes ${ }^{43}$.» L'état des connaissances porte à croire que l'influence des relations sociales sur la santé peut s'avérer aussi importante que celte du tabagisme, de l'hypertension, de l'obésité et du manque d'activité physique. D'ailleurs, certains facteurs de risque pour la santé fréquemment identifiés comme des constituants du mode de vie devraient plutôt être liés à l'environnement social.

42 L. Berkman et S.L. Syme, «Social Networks, Host Resistance, and Mortality; A Nine-year Follow-up Study of Alameda Country Residents», American Journal of Epidemiology, 109, 2, 1979, p. 186-204.

43 Institut canadien de recherches avancées, op. cit., p. 10. 
Le milieu de travail représente un aspect fondamental de l'environnement social d'un individu. Une étude britannique a montré que les conditions de travail et principalement la liberté de prendre des décisions contribuent à l'association très forte qui existe entre la classe sociale et les maladies cardiaques ${ }^{44}$. Les auteurs notent le fait suivant: moins la personne a de liberté de décision au travail, plus elle est portée à fumer. Robert Karasek et Tores Theorell, dans l'ouvrage Healthy Work: Stress, Productivity, and the Reconstruction of Working Life, s'intéressent aussi à l'influence de l'autonomie de décision au travail sur les symptômes de maladies cardio-vasculaires ${ }^{45}$. Dans la population observée, ces symptômes étaient très présents (20\%) chez ceux qui décrivaient leur fonction comme psychologiquement exigeante et ne permettant pas beaucoup d'autonomie de décision. Alors que les personnes pour qui le travail comportait une tension psychologique et qui possédaient une grande marge d'autonomie quant à l'utilisation de leurs compétences ne présentaient pas de symptômes de maladies cardio-vasculaires. De l'étude des liens entre la classe sociale et les maladies cardio-vasculaires chroniques ressort, selon l'ICRA, une conclusion primordiale: «Au-dessus d'un seuil de pauvreté, la place que l'on occupe dans la hiérarchie sociale peut être en soi un déterminant de la santé et de la maladie plus important que les conditions matérielles ${ }^{46}$.»

Les effets du chômage sur la santé ont aussi été étudiés. Une synthèse des travaux de recherche réalisée sous l'égide de l'Organisation mondiale de la santé révèle « qu'un taux élevé de chômage et l'instabilité économique entraînent une augmentation significative des problèmes de santé mentale et ont également des effets nuisibles non seulement sur la santé physique des chômeurs, mais également sur celle

44 M.G. Marmot et T. Theorell, loc. cit.

45 R. Karasek et T. Theorell, Healthy Work: Stress, Productivity, and the Reconstruction of Working Life, New York, Basic Books Inc., 1990.

46 Institut canadien de recherches avancées, op. cit., p. 10. 
de leur famille et de la collectivité 47 ». Une étude danoise portant sur le chômage et la mortalité a révélé un taux de mortalité plus élevé (entre 40 et $50 \%$ de plus) chez les chômeurs 48 . Cette étude, qui dura de 1970 à 1980, révéla un taux de mortalité plus important pour les principales causes de décès, en particulier pour les suicides et les accidents. De plus, dans les régions du Danemark présentant un taux de chômage plus élevé, le taux de mortalité parmi les chômeurs était moins élevé, comme si leur regroupement atténuait d'une certaine manière l'impact de leur situation.

Devant ces résultats, on pourrait soutenir que le lien entre la mauvaise santé et la pauvreté est un lien de causalité inversée. Ou, en d'autres mots, que les différences de mortalité par niveau de revenu peuvent être expliquées par le fait que les personnes malades deviennent pauvres et non pas que les pauvres deviennent malades. Mais les études longitudinales portant sur la santé des personnes vivant des changements de travail et de revenu démontrent que seule une part minime, environ 10\%, des différences observées entre santé et classe sociale/emploi peut être expliquée par le renversement du lien de causalité 49. Par exemple, l'étude réalisée au Danemark sur le chômage et la santé montre que l'écart ne s'est pas réduit au cours des dix ans que dura l'étude, alors que cela aurait dû se produire si, parmi les individus sans emploi, on avait seulement retrouvé, par autosélection, un grand nombre de personnes déjà malades.

47 G. Westcott et al. (sous la direction de), Health Policy Implications of Unemployment, Copenhague, World Health Organisation, 1985, p. 3.

48 L. Iverson, O. Anderson, P. Anderson et al., «Unemployment and Mortality in Denmark, 1970-80», British Medical Journal, 295,10 octobre 1987, p. 879884.

49 ICRA, op. cit., p. 11; R.G. Wilkinson, «Socio-economic Differences in Mortality: Interpreting the Data on their Size and Trends», dans: G. Wilkinson, Class and Health Research and Longitudinal Data, New York, Tavistock Publications, 1986, p. 1- 20. 


\section{CONCLUSION}

$\underline{\text { Retour à la table des matières }}$

L'existence d'une hiérarchie sociale est-elle inévitable? Oui, non, peut-être... Répondre à cette question ne constituait pas, bien sûr, l'objet de la présente réflexion. Nous avons tenté plutôt de vérifier si, à l'aube de l'an 2000, l'existence d'une hiérarchie sociale se traduit encore en inégalités sociales devant la maladie, la mort et les problèmes sociaux. Compte tenu des progrès technologiques récents et des investissements massifs dans les services de santé observés dans la plupart des pays industrialisés, analyser l'influence des facteurs sociaux sur la santé et le bien-être collectif nous semble avoir été riche d'enseignements. Nous avons vu qu'en dépit de l'existence de systèmes de soins et de services de qualité, plusieurs facteurs sociaux affectent encore la santé collective. L'influence à travers le temps du statut socioéconomique sur la santé a été comparée à une puissante rivière. $\mathrm{Si}$ vous identifiez les éléments de son parcours et tentez de bloquer sa course, elle trouvera simplement une nouvelle route pour atteindre sa destination 50 .

Mais au-delà de cette constatation, nous savons peu de chose sur la manière dont le social agit sur le biologique. Bien que plusieurs hypothèses explicatives fort intéressantes se dessinent à propos de la vulnérabilité liée à certaines situations sociales, il est déplorable de constater le peu d'investissements consacrés à la compréhension de l'influence des déterminants sociaux sur la santé et le bien-être des populations. Or, tout indique que les principaux gains de santé espérés par les collectivités résulteront vraisemblablement d'une meilleure com-

50 James S. House et al., loc. cit. 
préhension de cette relation. «Une compréhension complète de la relation entre la situation sociale, la capacité de faire face aux situations, le stress qui y est relié et la santé nécessitera des recherches au niveau plus` général de la population plutôt que de l'étiologie de maladies spécifiques ${ }^{51 . » ~ C o m m e ~ l e ~ s o u l i g n a i t ~ r e ́ c e m m e n t ~ l ' O r g a n i s a t i o n ~}$ mondiale de la santé, dans un document intitulé Santé et réformes économiques.

C'est en analysant les raisons de cette vulnérabilité et en élaborant des stratégies pour transformer ces situations que l'on parviendra à éliminer certains des obstacles majeurs au développement social, économique et politique. Les groupes vulnérables constituent eux-mêmes une ressource humaine que la plupart des stratégies de développement économique n'ont fait que marginaliser ${ }^{52}$.

Les travaux de recherche effectués dans les domaines de la santé, des services sociaux et de l'éducation démontrent l'importance de la petite enfance 53 . Les rapports entre la situation sociale connue dans l'enfance et le niveau d'instruction atteint sont étroitement liés aux problèmes sociaux et de santé vécus à l'âge adulte; la réussite ou l'échec scolaire devra retenir particulièrement notre attention. Si l'on veut briser la chaîne parents défavorisés/enfants en difficulté/ futurs adultes pauvres aux prises avec des difficultés d'adaptation sociale de même qu'avec de nombreux problèmes de santé, on se doit d'innover en matière de recherche afin de mieux comprendre les facteurs protecteurs et les facteurs de risques qui prévalent pendant l'enfance. Il faut établir quelles sont les conditions de vie, les attitudes et les valeurs familiales qui influencent négativement ou positivement l'adaptation sociale et la santé des enfants. Nous devons identifier les principaux

51 ICRA, op. cit., p. 9.

52 Organisation mondiale de la santé (OMS), Santé et réformes économiques, La santé, condition du développement économique, Genève, 1991, p. 64.

53 Wadsworth, M.E.J., The Imprint of Time. Chilhood, History and Adult Life, Oxford, Clarendon Press, 1991, 251 p. 
facteurs sociaux qui, au fil des années, expliquent la réussite ou l'échec scolaire d'enfants issus de familles défavorisées.

Au moment où le Québec, à l'instar de l'Ontario et de plusieurs États américains et européens, intensifie l'action préventive auprès des enfants pauvres ainsi qu'auprès de leur famille, le besoin d'innovation en matière d'intervention et de recherche s'avère capital. Un nouvel équilibre dans nos priorités s'impose. Nous devons dépasser la priorité consentie sans cesse à la recherche biomédicale et à la recherche en sciences naturelles pour accorder une plus grande importance à la compréhension des facteurs sociaux qui influencent l'état de santé et de bien-être collectif.

\section{BIBLIOGRAPHIE SÉLECTIVE}

$\underline{\text { Retour à la table des matières }}$

CALDWELL, J.C., «Routes to Low Mortality in Poor Countries», Population and Developpement Review, 12, 2, juin 1986, p. 171-220.

CHOINIÈRE, R., «Les disparités géographiques de la mortalité dans le Montréal métropolitain, 1984-1988: études écologiques des liens avec les conditions économiques, sociales et culturelles», Les cahiers québécois de démographie, 20, 1991, p. 117-146.

COHEN, S., «Social Support and Physical Illness», Advances, Institute for the Advancement of Health, 1990, 7, 1, p. 35-48.

COLIN, C., J.-P. LAVOIE et C. POULIN, Les personnes défavorisées, Et la santé, ça va?, Les publications du Québec, 1989, 119 p.

CURTIS, S.E., A. TAKET, R. PROKHORSKAS, M.A. SHABANAH et M.C. THURIAUX, «Vers la santé pour tous dans la région européenne de l'OMS. Surveillance des progrès accomplis. II. Condi- 
tions préalables: bilan démographique et sanitaire», Épidémiologie et Santé publique, 37, 1989, p. 295-317.

DESPLANQUES, G., «L'inégalité sociale devant la mort», Mortalité et causes de décès en France,

Collection «Grandes enquêtes en santé publique et épidémiologie», INSERM, France, \&, 1990, chapitre XII, p. 257-287.

HOUSE, J.S. et al., «Age, Socioeconomic Status, and Health», Milbank Quarterly, 68, 3, 1990, p. 384-411.

, «Social Relationships and Health, Science, 241, 29 juillet 1988, p. 540-545.

L'Institut canadien de recherches avancées, Les déterminants de la santé, publication $n^{\circ}$ 5, Toronto, Canada, septembre 1991, 39 p.

KARASEK, R. et T. THEORELL, Healthy Work: Stress, Productivity, and the Reconstruction of Working Life, New York, Basic Books Inc., 1990.

MARMOT, M.G. et G.D. SMITH, «Why Are the Japanese Living Longer?» British Medical Journal, 1989, 299, 23-30 décembre, p. 1547-1551.

MARMOT M.G. et T. THEORELL, «Social Class and Cardiovascular Disease: The Contribution of Work», International Journal of Health Sciences, 1988, 18, 4, p. 659-674.

McEVAN, Peter J.M., Editor-in-chief, «Special issue, Health inequities in Europe», Social Science and Medicine, 31, 3, 1990.

MECHANIC, D. et al., Pathways to Health: The Role of Social Factors, Menlo Park, Kaiser Foundation, 1989, p. 3-75.

Organisation mondiale de la santé (OMS), Santé et réformes économiques. La santé, condition du développement économique, Genève, 1991, 68 p.

PAMPALON, R., G. RAYMOND, D. BEAUDRY et D. GAUTHIER, La santé à la carte. Une exploration géographique de l'enquête Santé Québec, Les Publications du Québec, 1990, 60 p.

PAQUET, G., Santé et inégalités sociales. Un problème de distance culturelle, Québec, Institut québécois de recherche sur la culture, 1989, $131 \mathrm{p}$. 
Programme des Nations Unies pour le développement (PNUD), Rapport mondial sur le développement humain 1992, Paris, Economica, 1992, 229 p.

SMITH, G.D., M. BARTLEY et D. BLANE, «The Black Report on Socioeconomic Inequalities on Health Ten Years On», British Medical Journal, 301, 1990, p. 373-377.

SYME, S.L., "Control and Health: A Personal Perspective, Advances, The Journal of Mind-Body Health, 7, 2, printemps 1991, p. 16-27.

WADSWORTH, M.E.M., The Imprint of Time. Childhood, History, and Adult Life, Oxford, Clarendon Press, 1991, 251 p.

WHITEHEAD, M., «The Concepts and Principles of Equity and Health», Health Promotion International, 6, 3, 1991, p. 217-228.

WILKINS, R., O. ADAMS et A. BRANCKER, «Évolution de la mortalité selon le revenu dans les régions urbaines du Canada entre 1971 et 1986», Rapports sur la santé, Statistique Canada, mars 1990, p. 137-174.

WILKINSON, R.G., «Income Distribution and Life Expectancy», British Medical Journal, 304, janvier 1992, p. 165-168. 


\section{RÉSUMÉ}

\section{par Serge Cantin}

$\underline{\text { Retour à la table des matières }}$

In Traité des problèmes sociaux EN BREF. Synthèse préparée par Serge Cantin, Chapitre 11, pages 51-53, Sous la direction de Fernand Dumont, Simon Langlois et Yves Martin. Québec: Institut québécois de recherche sur la culture, 1995, 205 pages.

Au Québec, les services de santé accaparent près de $9 \%$ de la richesse collective, comparativement à $4 \%$ il y a trois décennies. À rebours de l'idée très répandue voulant que l'état de santé dépende du nombre de médecins et d'hôpitaux disponibles, nous découvrons l'importance cruciale des facteurs sociaux sur la santé, la maladie et la mort dans les sociétés industrialisées et notamment au Québec.

Dans un premier temps, on fait le point sur les conceptions de la santé et du bien-être. Depuis une cinquantaine d'années, l'équation «santé = soins de santé» a été largement admise, sans que l'on semble trop se préoccuper du fait que l'accroissement des dépenses de santé ne se traduisait pas nécessairement par une amélioration de la santé collective. Bien que cette perception étroite de la santé domine encore dans bien des milieux, une nouvelle conception, plus globale, de la santé est apparue au cours des dernières années qui prête attention non seulement aux dimensions psychologiques, mais également aux causes sociales de la maladie telles qu'elles se manifestent à travers les écarts de niveaux de santé observés entre différents groupes de la population. Certains de ces écarts, ceux qui relèvent par exemple de variations biologiques liées au vieillissement, sont inévitables; d'autres, par contre, renvoient à des facteurs évitables et peuvent être qualifiés d'injustes. Tels sont: a) les effets néfastes sur la santé de certains sty- 
les de vie, lorsque les possibilités de choisir sont sévèrement limitées; b) l'exposition à l'insalubrité et à des conditions de vie et de travail difficiles; c) l'accès inadéquat à des services de santé essentiels et à d'autres services publics. Dans cette dernière catégorie de facteurs qui peuvent, à des degrés divers, compromettre l'accessibilité égale aux services, on retrouve la pauvreté, la race, le sexe, l'âge, la religion; certaines barrières financières, organisationnelles et culturelles; une répartition inégale des ressources affectées aux services et aux soins de santé entre les régions; enfin, une trop large proportion de ressources consacrées à des services de haute technologie.

Dans un deuxième temps, on dresse un bilan des connaissances sur l'inégalité sociale devant la maladie et la mort. Dans presque tous les pays développés, des inégalités significatives ont été observées au plan de la santé entre les catégories les plus privilégiées et les moins favorisées de la société. De telles inégalités, qui se mesurent en termes d'espérance de vie, de mortalité, de prévalence de maladies spécifiques, etc., ne peuvent être liées qu'à des facteurs sociaux et culturels, puisque dans la plupart des sociétés modernes tous les citoyens ont également accès à un système de santé public. Des recherches menées au Canada confirment l'existence de ces inégalités entre classes sociales; par exemple, on y observe que l'espérance de vie d'un retraité est d'autant plus grande que son revenu aura été élevé pendant les douze années précédant sa retraite. Au Québec, même si dans l'ensemble la situation socio-sanitaire s'est améliorée, il n'en demeure pas moins selon les études - notamment l'Enquête Santé Québec - que les Québécois et Québécoises à faible niveau d'instruction et de revenu présentent plus de problèmes de santé et de problèmes sociaux ainsi que plus de décès prématurés que les autres. Pour l'espérance de vie en bonne santé, qui tient compte de la durée et de la qualité de vie, l'écart entre riches et pauvres serait de 14,4 ans. Un écart de 10 ans est également observé entre les espérances de vie des populations de quartiers favorisés et défavorisés de Montréal. Quant au taux de mortalité infantile, il est deux fois plus élevé en milieu défavorisé. Et ainsi de suite. Une relation a également été établie entre la pauvreté et les mauvaises habitudes de vie, de même qu'entre la pauvreté et l'incidence des problèmes sociaux. 
Dans un troisième temps, quelques hypothèses sont avancées concernant l'influence des facteurs sociaux sur la mortalité et la maladie. La stabilité des écarts de mortalité et de morbidité entre les classes socio-économiques sur une longue période de temps indiquent la présence de facteurs sociaux sous-jacents qui agissent au fil des générations. Deux facteurs s'avèrent déterminants. Le premier est la pauvreté. On reconnaît assez aisément l'existence de corrélations entre le produit national brut (PNB) per capita et l'espérance de vie. Mais des études récentes ont attiré l'attention sur la relation existant entre le degré d'équité dans la répartition de la richesse (la pauvreté relative) et l'état de santé. Ainsi, au Japon, l'espérance de vie à la naissance est passée entre 1955 et 1986 de 63,6 à 75,2 ans pour les hommes, et de 67,8 à 80,9 ans pour les femmes. Cette augmentation est liée de toute évidence à la croissance phénoménale du PNB japonais durant la même période, mais elle tient sans doute également au fait que l'écart relatif de revenu entre les plus pauvres et les $20 \%$ plus riches de la population japonaise est le plus faible de tous les pays de l'OCDE. Sur ce point, un rapport publié en 1992 par les Nations Unies montre que même si le Canada est l'un des pays qui offrent les meilleures conditions de vie, des disparités importantes y subsistent néanmoins et que de nombreux Canadiens et Canadiennes ne profitent guère des progrès du développement. Quant au Québec, sa situation générale s'est grandement détériorée au cours des dernières années au regard des différents indices de pauvreté (les personnes seules, les familles monoparentales, etc.), ce qui ne peut qu'entraîner des effets directs et indirects sur la santé et le bien-être des plus défavorisés. Le second facteur de risque majeur pour la santé et le bien-être est l'environnement social et plus particulièrement les conditions et le milieu de travail. Des études ont montré que la liberté de prendre des décisions, la place que l'on occupe dans la hiérarchie de l'entreprise et le niveau d'autonomie dont on dispose quant à l'utilisation de ses compétences, sont autant de variables qui entrent en jeu dans le lien très étroit que l'on observe entre la classe sociale et les maladies cardiovasculaires. Les effets du chômage sur la santé ont également été démontrés.

Dans la conclusion, on souligne et déplore la disproportion entre les sommes énormes investies dans la recherche biomédicale et le peu d'investissements consacrés à la compréhension de l'influence des déterminants sociaux sur la santé et le bien-être collectifs; alors que 
pourtant tout indique que les gains véritables dans le domaine de la santé ne résulteront que d'une meilleure compréhension de cette influence. L'action préventive auprès des enfants pauvres et de leur famille, dont on fait grand état aujourd'hui au Québec, n'aura d'efficacité qu'à cette condition.

\section{Fin du texte}

